\title{
Depression of the Aging Parents Who Lost Their Only Child in China
}

\author{
Peng Juan Wang ${ }^{1}$, Xiao Fu Pan ${ }^{1, ~ *, ~ J i n ~ Y u ~ L i u ~}{ }^{2}$ \\ ${ }^{1}$ School of Culture \& Social Development Studies, Southwest University, Chongqing, China \\ ${ }^{2}$ School of Social Work, University of Michigan, Ann Arbor, MI, USA
}

Email address:

cheerpxf@126.com (Xiao Fu Pan), pengjuanha@qq.com (Peng Juan Wang), jinyu.wuliu@gmail.com (Jin Yu Liu)

\section{To cite this article:}

Peng Juan Wang, Xiao Fu Pan, Jin Yu Liu. Depression of the Aging Parents Who Lost Their Only Child in China. American Journal of Applied Psychology. Vol. 4, No. 4, 2015, pp. 83-89. doi: 10.11648/j.ajap.20150404.11

\begin{abstract}
The CES-D scale is used to investigate depression of 201 aging parents who lost their only child in China. The results show that the bereaved parents have greater depression than the elder individuals in the general population. The results also indicate that sudden death and poor health have a significant positive relationship with depression of older parents who lost only one child. There is significant negative relationships between PTG and depression of older parents who lost only one child. The findings suggested the necessity of exploring psychological well-being of child-deceased elders and importance of developing interventions to assist these elders achieve posttraumatic growth from loss of their child.
\end{abstract}

Keywords: Posttraumatic Growth, Lost Only Child, Bereaved Parents

\section{Introduction}

Since the implementation of family planning as China's basic national policy in the late 1970s, "one child per couple" (called the "one-child policy") has been the pervasiveness in the People's Republic of China. In the Chinese population, $35.9 \%$ come from one-child family, $52.9 \%$ is one and a half-child in their family (Yu, 2007). The family planning has played very important role for China to control population pressure, but the rapid decline in the population may result in many serious social problems, such as gender imbalance, shrinking workforce, population aging, loss of independence for the elderly and so on. Especially, some families lost only-one child because of some accident and sudden death, his parents became lost independence for the elderly who lost their only child because of accidents or death, and the couple cannot re-birth children. By 2012, over one million families had lost their only child and it is estimated that 76,000 families will be added to this group each year, which means that the population of child-deceased families will continue to swell in the near future (Wu \& Dang, 2013).

The loss of a child can be a devastating experience (Sean \& Samuel, 2008), and losing only-one child is more misfortune. Especially, under the background of Chinese traditional culture and the social system constructed by family, child is only hope in a family, only-one childless means that the family has no offspring, and the parents have no one to depend on and will be alone in the world when they get old, they are not only independence losing for them, but also they have undergone grievous psychological trauma. It's maybe they are undergoing more depression, but some researchers of posttraumatic growth believe that there are some positive change in the bereaved parents who lost their child. Recent studies and reports in mass media raised concerns about risks of these "one-child" families. One particular concern is the likelihood that the parents would have complicated grief if their only child died, how they are undergoing trauma of losing only-one child, how about their depression, and whether or not they can get posttraumatic growth.

\section{Literature Review}

The elderly depression has become the second-largest mental disorders which only second to Alzheimer's disease. Because of the serious consequences of depression, the disease has been predicted as the second largest-disease which can disable people by WHO (Liao, 2010). The elderly depression can lead to serious mood disorders, physical function decline and increase risk of death, which is also bad for mental health and quality of life of the elder persons. The question of the elderly depression has attracted much attention. As China's aging population degree is deepening, more and more people have paid close attention to the elderly 
depression. Review of these previous studies, they mainly focused on the status of the elderly depression, influencing factors and intervention countermeasures, etc. There are plenty of studies to detect depression of the elderly, but the detection rate of the elderly depression varies, for example, Zhang Huping and Guan Siming (2000) investigated 218 aging people, the detection rate that the level of depression of mild, moderate, severe were separately $5 \%, 7.3 \%$ and $6.8 \%$ (Zhang, Guan, 2000). Petronella, et al. (2007) investigated 2850 aging people over 75, the prevalence of depressive symptoms was $31.1 \%$ ( Petronella, et al. 2007)). An Pan \& Franco (2007) surveyed on the 3289 50-70 year-old residents in Beijing and Shanghai and found that the incidence of depressive symptoms in the total study population was $9.5 \%$, the morbidity of female is higher than male $(6.7 \%$ for men and $11.7 \%$ for women). Zhou \& Chu (2008) found that the depression detection rate was $6.2 \%$ among 1600 elderly in Hunan province. Some studies showed that the depression detection rate of empty-nest elderly were significantly higher than those who were not, such as a research on the depression level of 100 empty-nest elderly in Beijing, 49\% of them had varying degrees of depressive symptoms, $10 \%$ of them had major depressive disorder, in which the occurrence rate of empty-nest elderly was higher for those who lived alone, with no medical insurance and had less contact with their children and relatives (Wang, Wang, 2013).

The elderly depression affected the normal quality of life of aging people seriously, such as, their depression scale scores was found negatively correlated with the quality of life of the aging people (Hou, Ma, 2013); the aging depressed patients were prominent of being anxiety, agitated, and they had memory loss, obvious physical symptoms, some of them even suicide. (Chen, $\mathrm{Xu}, 2012$ ).

The reasons of the elderly depression vary, such as psychological and physiological hypo-function, loneliness, family dissension, valetudinarianism, economic difficulties such factors can cause or contribute to the occurrence and expedition of geriatric depression (Mo, 2008). Rural and urban factors have significant effect on the elderly depression, depression of the aging people in rural areas was significantly higher than the aging people in urban (Pan, Wang, 2012). On the one hand, the economy in rural was lower than urban (Chen, XU, 2012), on the other hand, the urban people paid more attention to the mental health than rural areas. Gender and age affected the level of elderly depression significantly, female incidence of depression and the average level of depression was higher than male (Pan, Wang, 2012; Zhang, Guan, 2000; Zhou, Chu, 2008 etc.). With the growth of age, the elderly depression was significantly enhanced (Pan, Wang, 2012; Chen, XU, 2012 etc.). Education level also has a significant impact on the elderly depression, the higher the education level, the lower level of the elderly depression ( (Pan, Wang , 2012; Chen, XU , 2012 etc.). Child was also an important factor influencing the elderly depression, children were the most important social support on emotional, financial, information for elder people (Brubaker, 1990; Stoller and Pugliesi, 1991; Beggs et al., 1996; McMullin and Marshall,
1996), the aging people without children had much higher levels of depression than who had children (Pan, Wang, 2012; Liu, Guo, 2008). At the same time, social support, economic status (Deng, Gan, 2008; Wu, Sun, 2010; Dr K.-L. Chou, A. H Y. Ho, 2006, Neal Krause , Jersey Liang, 1998), health status, self-care in y life (Chen, XU, 2012), lifestyle (Hua, Hua, 2012), self health statement (Ada CMui, 1996) have also been discussed to be a negative correlation with the elderly depression.

For the elderly depressive symptoms, many scholars put forward the corresponding intervention countermeasures. For example, Ian JN (1997) proposed a system of preventive measures, which includes three stages: the early prevention, secondary prevention, and prevention in the end. Zhang Hong (2005) advocate the use of psychotherapy, drug therapy, physical exercise therapy, electric convulsive therapy (ECT) and other treatments to relieve the symptoms of elderly depression. Jin Yingying (2011) proposed to reduce the occurrence of depression prevention, improve the quality of life of older people, and promote the health of the elderly, then reduce health care costs and mortality by the home care for the elderly. Zhu Li-chang \& Hua Ying xue (2011) proposed to improve the social pension system, deepen the reform of community health service model agency, establish and improve the community's elderly services, and strengthen child care elderly, establish and improve community elderly human health services, improve the aging people's health, thereby improving their psychological from the macro level.

Although there were many researches on depression in the aging people at home and abroad, but the concern on depression of Chinese aging people who lost their only child was few, studies remained in the dilemma analysis of the aging people who lost their only child, for example, Liu Zhiyan (2012) analyzed the problems they were facing from the spiritual, social, psychological, and physical four aspects, and thought that the death of children made their parents exhausted both in body and mind, the decline of physical health and the rise of psychological problems, unable to carry out the normal social contact. Mu Guangzong (2011) divided the crisis of the families which lost only child into investment losses, emotion losses and utility losses. Zhao Zhongjie (2009) had the same opinions on the metal shock and pension deficiency for families which lost only child, but he added the analysis angle of Family Disintegration and believed that children were the link for the spousal relationship. If the only child dead unfortunately, the link broke and family structure crushed, the spousal relationship would have high pressure and cause family disintegration for the divorce. Besides the influences above, Xu Jimin (2007) believed the death of only child also could cause the parents to work carelessly, have revenues, closed, desocialized, their entire social life would be disorganized, they would not want to see a stranger and appear serious psychological problems, even suicide. It was a pity that there was only few specialize in the depression of the aging people who lost their only child, such as Fang Shuguang (2013) proposed most of them had depressive disorders. Because they suffered more, they were more depressed, 
worried and desperate equally, etc. (Yu, 2013) Only $20.0 \%$ to $25.0 \%$ of normal old people had depression, impetuous, sadness, loneliness and other negative emotions, the negative emotions existed more common in the elderly people who lost their only child, a lot of them wanted to contact with society no longer, there are more fragile and sensitive in mentality, and they even chose to self-reclusive, so compared with material support, it was extremely urgent to comfort their mentality ( $\mathrm{Li}, \mathrm{He}, 2013$ ). According to the survey, $63.3 \%$ of the families which lost the only child showed they Don't want to go out, $50.2 \%$ of them showed they Don't want to speak to people used to know, and they were afraid to talk with topics involved with their children (Chen, 2012). Although researches above had mentioned the depression problem of the elderly people who lost their only child, there were no actual measurement on the depression levels of them. The depression levels of assessment results of this research will be helpful to know more about them, appeal the society to pay attention to this group positively, and provide related policy basis for the government.

\section{Methods}

\subsection{Participants}

This study used the cluster sampling method, enrolled 205 families that parent lost their only-one child from Beibei district of Chongqing municipality, China. Each family randomly chose one to response. At last, 205 participants take part in survey. Inclusion criteria: the participants should be aged 49 years and or above, and their only child has been lost and the couple cannot re-birth children; willing to participate in; could communicate in Chinese; primary school level or higher complete the test by themselves, and illiterate participants finished the test by interpretation of training research assistants (RAs).

\subsection{Measures}

\subsubsection{General Information Questionnaire}

A self-compiled questionnaire, consists of demographic data like age, gender, dwelling place, educational level, marital status, self-perceived economic status, The reason of losing child, status of health, and status of social support.

\subsubsection{The CES-D Scale}

The Center for Epidemiologic Studies Depression Scale (CES-D Scale) was developed by Lenore Sawyer Radloff (1977) is used in studies of the epidemiology of depressive symptomatology in the general population. It includes 20 items in the scale, respectively investigating 20 symptoms. According to the degree in the past week in corresponding situation or feeling the frequency of evaluation to respond. " $0=$ never"," $1=$ sometime have"," $2=$ often have"," $3=$ always have". Computed the total score, the score 15 or less $=$ no depression symptoms, the score $16 \sim 19=$ have some depression symptoms, the score 20 or more $=$ have severe depressive symptoms. Many studies have demonstrated it has good reliability and validity. In the current study, Cronbach's alpha is 0.897 .

\subsubsection{The PTG Scale}

PTG is evaluated by The Posttraumatic Growth Inventory Revision (PTGI-R); PTGI-R is based on PTGI (Tedeschi \& Calhoun, 1996), 21-items are translated into Chinese by researcher who good at English language. Through the test of exploratory factor analysis, we got 18-item inventory that assesses five domains including new possibilities, relating to others, personal strength, spiritual change, and appreciation of life. The result of confirmatory factor analysis shows that $\chi^{2}=290.24, \mathrm{df}=125, \chi^{2} / \mathrm{df}=2.32, \mathrm{RMSEA}=0.078, \mathrm{NFI}=0.92$, $\mathrm{NNFI}=0.94$, RFI $=0.90, \mathrm{CFI}=0.95$, GFI $=0.87$, So PTGI-R has demonstrated adequate construct validity in this study, with individuals who higher score denoting more posttraumatic growth. In the current study, Cronbach's alpha is adequate for total score (0.87) and specific subscales(0.70 to 0.87$)$.

\subsection{Procedure}

Based on the sample size, multiple research assistants were used in data collection, to establish inter-rater reliability for GDS and PTG, training content of RAs contained: unifying explanation oral before assessment; unifying standard for evaluation. Then, during our door-to-door field survey, the trained RAs distributed the questionnaires to each participant and told or helped them on how to complete the instruments; face-to face interviews were conducted in the subjects' homes and took 50 minutes on average. This study continued from 2013 May to 2013 September. Finally, 201 participants completed the questionnaire thoroughly. The response rate of questionnaires was $98.05 \%$.

\section{Results}

\subsection{Descriptive Result of all Outcome Variables}

Table 1. descriptive results $(N=201)$.

\begin{tabular}{lll}
\hline & Mean (\%) & SD \\
\hline Age (in years, range: 47-80) & 60.84 & 7.18 \\
Gender (\%) & 52.24 \\
Male & 47.76 \\
Female & \\
Marital status (\%) & 66.00 \\
Married & 34.00 \\
Widowed or divorced & \\
Rural/urban residence (\%) & 36.82 \\
Living in rural village & 63.18 \\
Living in urban community & \\
Self-evaluation of living standard (\%) & 44.28 \\
Good or neutral living standard & 23.38 \\
Poor living standard & 32.34 \\
Very poor living standard & \\
Education (\%) & 42.29 \\
Illiterate or elementary school & 35.82 \\
Junior middle school & 21.89 \\
High school and higher & \\
Self-rated health status (\%) & 20.00 \\
Good health & 19.44 \\
Neutral health &
\end{tabular}




\begin{tabular}{lll}
\hline & Mean (\%) & SD \\
\hline Poor health & 37.78 & \\
Very poor health & 22.78 & \\
Length of grief (in years, range: 0-41) & 9.27 & 6.26 \\
Reason of child's death (\%) & & \\
Sudden accident & 50.25 & \\
Non-sudden & 49.75 & \\
Post trauma growth (range: 24-89) & 67.73 & 11.27 \\
Depression (range: 0-60) & 23.81 & 8.56 \\
Severe depression & 71.6 & \\
Medium depression & 14.00 & \\
Never depression & 15.40 & \\
\hline
\end{tabular}

Table 1 shows that in the valid samples, including 105 males (52.24\%)and 96 females(47.76\%); The mean age of the respondents was 60.84 and $61.38 \%$ were living in urban communities; $66 \%$ is married, $34 \%$ is widowed or divorced; $42.29 \%$ is level of illiterate or elementary school, $35.82 \%$ is level of junior middle school and $21.89 \%$ is level of high school and higher; $44.28 \%$ of Self-evaluation of living standard is good or neutral, poor and very poor is respectively $23.38 \%, 32.34 \%$; Self-rated health status of good health is $20 \%$, of neutral health is $19.44 \%$, of poor health is $37.78 \%$, of very poor health is $22.78 \%$; The mean length of grief is 9.27 years. Severe depression is $71.6 \%$, medium depression is $14 \%$, only $15.4 \%$ is never depression

\subsection{The Comparison Between Detection Rate of Depression of the Elder Parents Who Lost Their Only Child in China and Those Who Have Several Children}

15273 aging individuals who have several children are chosen from China Family Panel Studies (CFPS) to compare with 201 elder parents who lost their only child in China. Comparing the difference between the detection rate of their depression, the results show as Table 2.

Table 2. the chi-square test between detection rate of depression of the elder parents who lost their only child in China and those who have several children.

\begin{tabular}{llll}
\hline & $\begin{array}{l}\text { Detection rate of depression of the elder parents } \\
\text { who lost their only Child in China (\%) }[\mathbf{n}=\mathbf{2 0 1}]\end{array}$ & $\begin{array}{l}\text { Detection rate of depression of the elder parents who } \\
\text { do not lost their only child in China (\%) [n=15273] }\end{array}$ & $\begin{array}{l}\text { Chi-square } \\
\text { value } \mathbf{P}\end{array}$ \\
\hline Never depression & 15.40 & 67.6 & $0.000^{* * * *}$ \\
Medium depression & 14.00 & 26.8 & 6.6 \\
Severe depression & 71.60 & & 6 \\
\hline
\end{tabular}

Table 2 shows that the chi-square test between detection rate of depression of the elder parents who lost their only child in China and those who not. From it, we know, it is significant different between them. Detection rate of depression of the elder parents who lost their only child in China is significantly higher than those who not.

\subsection{Linear Regression Models of Depression in the Elderly Who Lost Only One Child in China}

Table 3. Linear regression models of depression in the elders who lost their only child.

\begin{tabular}{llll}
\hline & MODEL1 & MODEL2 & MODEL3 \\
\hline Age & -0.104 & -0.048 & -0.069 \\
Gender & $(0.095)$ & $(0.094)$ & $(0.094)$ \\
& -1.082 & -1.036 & -0.565 \\
married & $(1.368)$ & $(1.350)$ & $(1.350)$ \\
& -1.902 & -1.768 & -2.046 \\
rural & $(1.391)$ & $(1.374)$ & $(1.362)$ \\
& -0.255 & 0.533 & 0.304 \\
Self-evaluation of living standard & $(1.568)$ & $(1.603)$ & $(1.587)$ \\
Poor living standard & -0.792 & -0.916 & -0.360 \\
& $(1.755)$ & $(1.744)$ & $(1.740)$ \\
Very poor living standard & 1.109 & 0.870 & 1.494 \\
& $(1.817)$ & $(1.810)$ & $(1.809)$ \\
Education & & & \\
Junior middle school & -2.108 & -1.608 & -1.871 \\
& $(1.598)$ & $(1.597)$ & $(1.582)$ \\
High school and higher & $-3.989^{*}$ & -2.922 & -2.410 \\
& $(1.972)$ & $(1.971)$ & $(1.960)$ \\
Self-rated health status & & & \\
Neutral health & 1.858 & 2.138 & 2.476 \\
& $(2.092)$ & $(2.075)$ & $(2.055)$ \\
\hline
\end{tabular}

\begin{tabular}{llll}
\hline & MODEL1 & MODEL2 & MODEL3 \\
\hline Poor health & $4.533^{*}$ & $4.528^{*}$ & $4.144^{*}$ \\
& $(1.880)$ & $(1.866)$ & $(1.851)$ \\
Very poor health & $4.893^{*}$ & $5.303^{*}$ & $4.999^{*}$ \\
& $(2.212)$ & $(2.208)$ & $(2.185)$ \\
Length of grief & & -0.006 & -0.008 \\
& & $(0.102)$ & $(0.101)$ \\
Sudden death of the only kid & & $3.892^{* *}$ & $3.296^{*}$ \\
& & $(1.288)$ & $(1.300)$ \\
Post trauma growth & & & $-0.131^{*}$ \\
& & & $(0.058)$ \\
Constant & $30.125^{* * *}$ & $23.943^{* * *}$ & $34.287^{* * *}$ \\
& $(6.214)$ & $(6.513)$ & $(7.901)$ \\
$R^{2}$ & 0.13 & 0.17 & 0.19 \\
$N$ & 180 & 178 & 178 \\
\hline \multirow{2}{*}{ Standard errors in parentheses; ${ }^{*} p<0.05,{ }^{* *} p<0.01,{ }^{* * *} p<0.001$}
\end{tabular}

Table 3 shows the results of linear regression analysis. We explored the relationship between depression and three sets of independent variables, demographic variable (including to characteristics of traumatic event, personal resources, and social support and so on), posttraumatic growth, separately from Model 1 to Model 3. The results of Model 1 indicate that Self-rated health status ( $\beta$ poor health $=4.533$, $\beta$ very poor health $=4.893, \mathrm{p}<.01)$ and high school and higher $(\beta=-3.898$, $\mathrm{p}<.01)$ were significantly related to depression. In Model 2, sudden death of only one child $(\beta=3.892, \mathrm{p}<.01)$ and poor health $(\beta$ poor health $=4.528, \beta$ very poor health $=5.303$, $\mathrm{p}<.01)$ are significantly positive related to depression. In Model 3, when other independent variables were controlled. The relationship between depression and posttraumatic growth was significantly negative $(\beta=-0.131, \mathrm{p}<.05)$, and sudden death of only one child $(\beta=3.296, p<.01)$ and poor 
health $(\beta$ poor health $=4.144, \beta$ very poor health $=$ $4.999, \mathrm{p}<.01)$ are significantly positive related to depression.

\section{Discussion}

The depressed condition of the aging parents who lost their only child of this research is as follows:

The percentage of severe depression is $71.6 \%$, medium depression is $14 \%$, only $15.4 \%$ never depress, and the depression of the aging parents who lost their only child is significantly higher than who don't( $\left.M=23.81, \chi^{2}=1395.92\right)$. Compared to other aged, the accidental death of the only child made the elderly parents exhausted both physically and mentally, even generated the decline of physical health and mental problems (Liu, 2012), that was why the depressed condition of the aging parents who lost their only child was much higher.

Children are the future hope of parents in Chinese family, the only child plays a more important role in family structure for the uniqueness. The accidental death of the only child has left trauma for their parents which is hard to heal, as well as psychic pain for their parents to undertake for a long-term, which make the parents more vulnerable in mental states and emotions, so the morbidity of mental illness such as depression of the aging parents who lost their only child is much higher than the others. Due to the particularity and complexity of living conditions of the aging parents who lost their only child, if they are depressed, the treatment and recovery for them will be more difficult. The society should establish long-term intervention mechanism and makes full use of resources around the aging parents who lost their only child, and strengthen mental health support for them, make efforts to resolve psychological barriers of them.

This research discovers that social support, educational statue, self-rated health level and health conditions have significant correlation with the depression of the aging parents who lost their child. Chen and Xu (2012) believed that social support, educational statue and health conditions are the main factors which influenced the depression of the aging people, and the most one was social support. The social support made great difference for the mental health of the aging people, especially for depression. (Deng, Gan,2008; Wu, Sun,2010; Dr K.-L. Chou, A. H. Y. Ho, 2006; Su, Wu, 2012). Some researches believed social support had regulating action for the arise and development of depression (Bow ling A, 1998; P rierce R, Fron EM, 2000; Xie, Zhang, 2009). To establish social support network had become a main measure to solve the depression problem for the aging people. (Fang, 2013; Yu, 2013; Li, He, 2013; Zhu, Hua, 2011 etc.). If the aging people had active self-health statement and high self-rated health level, they would reduce the morbidity of depression at minimum level (Ada CMui, 1996). The morbidity of depression of the aging people who had body disease and cerebrovascular disease was obviously higher than the health ones ( $\mathrm{Li}, \mathrm{Li}, 2006)$, the physical health had direct influence on the mental health of the aging people who lost their only child.

The social support network refers that people can acquire emotional support, material assistance and service, information and new social contact through personal contact. According to the social support theory, the stronger social support network a person has, the better he/she can deal with the varieties challenges of the environment. The resources for individual can be divided to personal resources and social resources, the personal resources include ego function and coping capacity, social resources refer to the breadth of personal social network and the degree of the social support that people can support in the network. Therefore, we need to integrate the government, social organizations, community, social workers and volunteers, and the own strength of the aging people, expand the scope and the content of social support for the elderly, and those are better ways to unite the social capital, and improve their confidence and abilities for facing life, then to reduce the depression of the elderly.

This research result shows that it has significant negative correlation between the depression level and posttraumatic growth. This result is in line with the past research results. For example, Yang Yaping and Zhang Zhenxiang (2013) found that it had significant negative correlation between the depression level and posttraumatic growth. Depression could cause painful emotional states, and it would be accompanied by a series of physical discomfort, those discomforts aggravated the individual's psychological burden in return. This was likely to add depressive symptoms of the elderly parents who lost their only child. Depression was a kind of negative mood, severe symptoms of depression could lead the patients to feel depressed, pessimistic and meaningless, even suicide. It was obviously that the depressive emotion went against for the post-traumatic growth for the elderly parents who lost their only child.5.3. The Limitation and Prospect of this Study.

The results of this study provide some theoretical support and practical guidance to lower levels of the depression of the aging people who lost their only child. According to the results of the research, we give the following suggestions: First, from the social support helps to reduce the depression of the aging people who lost their only child (Wang Xing-hua, etal., 2006), Community should increase the intensity of care of the aging people who lost their only child, attaches great importance to depression problems of the aging people who lost their only child, construct social support system. It ensures that the aging people can improve their life quality and spend their twilight years. Second, we can reduce the occurrence of depression of the aging people who lost their only child by community care. We can make full use of the advantages of community to strengthen the community care of the aging people who lost their only child. The government should develop programs, policies and regulations, personnel training, funding, community planning and construction of its guarantee to improve the level of scientific and standardized. At the same time, relatives and community residents should develop the tradition of respecting the aging people and establish a community care professional system to help the aging people who lost their only child. Third, the aging people who lost their only child 
should keep the peace of their mind, make friend with others, and strengthen their physical training to their psychosomatic health. Finally, this study also has some limitations: First, although the partial sample helps to understand the status of the depression of the aging people who lost their child, the date from beibei of Chongqing also has some representative problem of sample and regional cultural differences, which makes the conclusions of this study has certain limitations. Second, this research mainly using cross-sectional survey and the lack of longitudinal tracking cannot avoid the problems of ecological validity. Third, in the process of collecting data, part of the aging people who lost their only child didn't want to look back on the sadness of the past, as a result, the research data may have some deviation. In order to understand the depression status of the aging people who lost their only child, researchers should expand the sample of research in the future, and use the horizontal and vertical methods such as tracking study and cross-sectional study to enhance the ecological validity of the researches.

\section{Conclusion}

The findings from this study are as follows: Firstly, the bereaved parents have greater depression than the elder individuals in the general population. Secondly, the sudden death and poor health have a significant positive relationship with depression of older parents who lost only one child. Thirdly, there is significant negative relationships between PTG and depression of older parents who lost only one child. The findings suggested the necessity of exploring psychological well-being of child-deceased elders and importance of developing interventions to assist these elders achieve posttraumatic growth from loss of their child.

\section{Acknowledgements}

The authors would like to thank the reviewers for their helpful comments and suggestions on this research. This work was substantially supported by a grant from the National Social Science Found Project (11XSH019), and the Fundamental Research Funds for the Central Universities (SWU1409264) and (SWU1309371).

\section{References}

[1] Zhu L.Ch., Hua Y.X., Li L., et al. Mental Condition of Empty Nest Elders in Shanggang Community and Invention Strategy, Behavior • Social Psychology, 2011, 3

[2] Chen Zh., Xu X.J.. Depression in the Empty Nest Elderly in China and Psychological Intervention Program. Journal of Hubei University of Education. 2012,12.

[3] Li D.D., Zang N., He P.L., Cui R.R., Wei Zh. Zh., Yuan H.. Depression prevalence status and the influencing factors in empty nest elderly in a town of Anhui province. Journal of Wannan Medical College. 2013;32(1). 1002 -0217(2013)01 $-0079-03$.
[4] Wang Y., Wang X.L.. The relationship between receiving community care status and depression among empty nester in a community in Beijing. Research Paper. 2013.06.009.

[5] Zhou Ch.Ch., Chu J., Xu X.Ch, et al. Survey on Depression of Empty NestAged People in Rural Community of Anhui Province. Chinese Mental Health. Journa, 1 Vol 22, No.2, 2008.

[6] Zhang H.P., Guan S.M., Zhang Y.H.. Study on Depression in Aged in-patients in General. Department. Acta Univ Med Tongji.Vol.29 No.6 P.594 Dec.2000.

[7] Deng P., Gan W., Liu W.F., Xie T., Peng G.G., Li S.J. The Depression Conditions among Old People in Some Community and the Influential Factors. Journal of Nursing (China). No.2008.Vol.15 No.11.

[8] Mo K.J.. The senile despondent reason analyzes and nurses the countermeasure. The medicine leads the publication.2008.2.

[9] Zhang H.. Geriatric Depression adverse consequences and Intervention Measures. Ournal Of NingBo University. VoL. 1 27 NO. 4.Aug. 2005.

[10] Hua Y., Hua Q.Zh., Xu Sh.Sh., et al. Analysis the correlation between lifestyle and depression symptom of elderly. Nursing Practice and Research. 2012. 21.003.

[11] Petronella J. (Nelleke) van't Veer-Tazelaar, Harm W.J. van Marwijk. Depression in old age (75+), the PIKO study. Journal of Affective Disorders 106 (2008) 295-299.

[12] Pan A., Franco OH, Wang YF, Yu ZJ, Ye XW, Lin X. Prevalence and geographic disparity of depressive symptoms among middle-aged and elderly in China. Journal of Affective Disorders 105 (2008) 167-175.

[13] Ada, CMui. Depression among Elderly Chinese Immigrants: An Exploratory Study. National Association of Social Workers. Vol 41, Number 6 November 1996.

[14] Beggs JJ, Haines VA, Hurlbert JS. 1996. Situational contingencies surrounding the receipt of informal support. Soc Forces 75:201-222.

[15] Brubaker TH. 1990. Families in later life: a burgeoning research area. J Marriage Fam 52: 960-981.

[16] Stoller EP, Pugliesi KL. 1991. Size and effectiveness of informal helping networks: a panel study of older people in the community. J Health Soc Behav 32: 180-191.

[17] McMullin JA, Marshall VW. 1996. Family, friends, stress and well-being: does childlessness make a difference. Can J Aging 15:355-373.

[18] Li L., Li J.M., Li Zh.J.. The Relationship between onset of Senile Depression and Life Events. China Journal of Health Psychology. 2006, Vol 14, No. 4.

[19] Bow ling A.Social networks health and emotion well-being among the oldest old in London. $J$ Geronto, $l 1998$; 46( 7): 20-32.

[20] P rierce R, Fron EM, RussellM, et al. A longitudinal model of social contact, social support, depression, and alcohol use. Health Psycho, 12000; 19(1): 28-38.

[21] Xie L.Q., Zhang J. P.. Study of Rural empty nesters depression and social support, coping relations. Chinese Journal of Gerontology. 2009, 10(19): 2515-7. 
[22] Li Y.M., Chen Ch.Sh, Tu H.b.. Prevalence and risk factors for depression in older people in $\mathrm{Xi} 0$ an China: a community-based study. RESEARCH ARTICLE. Int J Geriatr Psychiatry 2012; 27: 31-39.

[23] Dr K.-L. Chou, A. H. Y. Ho,I. Chi. Living alone and depression in Chinese older adults. Aging \& Mental Health. November 2006; 10(6): 583-591

[24] Wu Zh. Q., Sun L., Sun Y.H.. Correlation between loneliness and social relationship among empty nest elderly in Anhui rural area, China. Aging \& Mental Health. Vol. 14, No. 1, January 2010, 108-112.

[25] Liu L.J., Guo J.. Life satisfaction in a sample of empty-nest elderly: a survey in the rural area of a mountainous county in China. Qual Life. Res (2008) 17:823-830

[26] Krause, Jersey Liang. Financial Strain, Received Support, Anticipated Support, and Depressive Symptoms in the People's Republic of China. Psychology and Aging[J].1998, Vol. 13. No. $1,58-68$.

[27] Su D., Wu X.N., etc. Depression and social support between China' rural and urban empty-nest elderly. Archives of Gerontology and Geriatrics. 55 (2012) 564-569.

[28] Zhang H., et al.. Adverse consequences and Intervention
Strategies of depression in elderly. J OURNAL OF N I NGBO UN I VERSI TY (EDUCAT I ONAL SCI ENCE)[J]. Vol27 NO.4 Aug.2005.

[29] Jin Y.Y.., et al. Etiology and home care of depression in elderly. CHINA FOREIGN MEDICAL TREATMENT. 2011 NO.20.

[30] Liao H.. The characteristics of elderly depression and its influence on the quality of life. Chinese Journal of Geriatric Care. 2010 (8).

[31] Wang Y., Wang X.L.. The relationship between receiving community care status and depression among empty nester in a community in Beijing. Chinese Nursing Management. 2013(6).

[32] Wang X.H., Wang D.H., Shen J.L.. The Effects of Social Support on Depression in the Aged. Chinese Journal of Clinical Psychology. Vol.14 No. 12006.

[33] Liu Zh. Y. Courage to live-loss of independence by calling for social concern groups. Theory Research. 1002-2589 (2012) 20-0057-02.

[34] Mu G.Z.. Only-child Families Are Essentially Risky Families. China Social Science Daily. 2011-11-03(A07).

[35] Yang Y.P., et al.. Studying on the negative correlation among anxiety, depression and posttraumatic growth for stroke patients. China Health Management. 2013 (02). 